\title{
BMJ Open Challenges and barriers to optimising sedation in intensive care: a qualitative study in eight Scottish intensive care units
}

\author{
Kalliopi Kydonaki, ${ }^{1}$ Janet Hanley, ${ }^{1}$ Guro Huby, ${ }^{2}$ Jean Antonelli, ${ }^{3}$ \\ Timothy Simon Walsh, ${ }^{3}$ on behalf of the Development and Evaluation of Strategies \\ to Improve Sedation practice in inTensive care (DESIST) study investigators
}

To cite: Kydonaki K, Hanley J, Huby G, et al. Challenges and barriers to optimising sedation in intensive care: a qualitative study in eight Scottish intensive care units. BMJ Open 2019;9:e024549. doi:10.1136/ bmjopen-2018-024549

- Prepublication history and additional material for this paper are available online. To view, please visit the journal (http:// dx.doi.org/10.1136/bmjopen2018-024549).

Received 6 June 2018 Revised 23 January 2019 Accepted 13 March 2019

Check for updates

(c) Author(s) (or their employer(s)) 2019. Re-use permitted under CC BY-NC. No commercial re-use. See rights and permissions. Published by BMJ.

${ }^{1}$ School of Health and Social Care, Edinburgh Napier University, Edinburgh, UK ${ }^{2}$ Faculty of Health and Social Studies, Østfold University College, Halden, Norway ${ }^{3}$ Department of Anaesthesia, Critical Care, and Pain Medicine, University of Edinburgh, Edinburgh, UK

Correspondence to Dr Kalliopi Kydonaki; C.Kydonaki@napier.ac.uk

\section{ABSTRACT}

Objectives Various strategies to promote light sedation are highly recommended in recent guidelines, as deep sedation is associated with suboptimum patient outcomes. Yet, the challenges met by clinicians in delivering highquality analgosedation is rarely addressed. As part of the evaluation of a cluster-randomised quality improvement trial in eight Scottish intensive care units (ICUs), we aimed to understand the challenges to optimising sedation in the Scottish ICU settings prior to the trial. This article reports on the findings.

Design A qualitative exploratory design: We conducted focus groups $(\mathrm{FG})$ with clinicians during the preintervention period. Setting and participants: Eight Scottish ICUs. Nurses, physiotherapists and doctors working in each ICU volunteered to participate. FG were recorded and verbatim transcribed and inserted in NVivo V.10 for analysis. Qualitative thematic analysis was undertaken to develop emergent themes from the patterns identified in relation to sedation practice. Ethical approval was secured by Scotland A Research ethics committee.

Results Three themes emerged from the inductive analysis: (a) a recent shift in sedation practice, (b) uncertainty in decision-making and (c) system-level factors including the ICU environment, organisational factors and educational gaps. Clinicians were challenged daily to manage agitated or difficult-to-sedate patients in the era of a progressive mantra of 'just sedate less' imposed by the pain-agitation-delirium guidelines.

Conclusions The current implementation of guidelines does not support behaviour change strategies to allow a patient-focused approach to sedation management, which obstructs optimum sedation-analgesia management. Recognition of the various challenges when mandating less sedation needs to be considered and novel sedationanalgesia strategies should allow a system-level approach to improve sedation-analgesia quality.

DESIST registration number NCT01634451

\section{INTRODUCTION}

Sedation and analgesia are provided in critically ill patients to increase compliance with invasive and uncomfortable procedures
Strengths and limitations of this study

- This study used a qualitative design to enable an in-depth exploration of the challenges that clinicians face when managing sedation and analgesia in critically ill patients in an era that less sedation is mandated through the pain-agitation-delirium international guidelines.

- This study provided a comprehensive insight of critical care context-specific challenges that need to be considered in future intervention research that aims to improve sedation-analgesia quality.

- A strength of the study was the achievement of maximum variation of roles and experience of the participants, which provided a rich insight into their perceptions.

- A weakness of the study is that the challenges presented related to Scottish intensive care units, and may differ to other national and international settings; hence, the findings cannot be generalised.

and to prevent or alleviate pain, discomfort, anxiety and stress. Recent guidelines, ${ }^{1}$ recommend the avoidance of deep sedation as it is associated with poorer physical and psychological outcomes and recovery among survivors. ${ }^{2}$ To achieve this, strategies that promote lighter sedation, such as nurse-led sedation protocols, daily sedation breaks and analgesia-based strategies, have been introduced and widely implemented recently, but may increase patient agitation, discomfort and adverse events. ${ }^{2}$ Reported challenges by clinicians using these strategies are the lack of coordination of sedation interruptions with the physicians' schedules mainly in university hospitals, ${ }^{3}$ cultural changes towards intracollegial openness, ${ }^{4}$ nurses' concerns of patient safety, the lack of evaluation of sedation protocols in difficult-to-sedate patients such as in neuroscience, trauma and substance abuse $^{5}$ and lack of consensus regarding the 
recommendations in guidelines. ${ }^{6}$ Although positive outcomes have been demonstrated, ${ }^{1}$ little consideration and description has been given to the contextual factors and intensive care unit (ICU) culture that could have affected the implementation and sustainability for change practice.

Between 2013 and 2015, we conducted a cluster-randomised quality improvement trial in eight Scottish ICUs to evaluate the effectiveness of three interventions for improving sedation-analgesia quality in mechanically ventilated (MV) patients; a bespoke education package on sedation-analgesia management, sedation-analgesia quality feedback measures and a novel sedation monitoring technology to detect deep sedation. ${ }^{78}$ During the preintervention phase, we aimed to explore clinicians' perceptions of challenges in achieving optimum sedation in the participating ICUs. This article reports a detailed inductive analysis of the data.

\section{METHODS}

We used a qualitative exploratory design to examine ICU clinicians' perceptions of challenges to optimum sedation care. We selected ICUs in Scotland from eight hospitals that admitted MV patients to represent a typical UK case mix. The nurse-to-patient ratio was 1:1 for MV patients, consistent with UK national guidance. We excluded ICUs that only admitted patients under the care of specialist cardiac, neurosurgical or paediatric teams.

We conducted focus groups (FG) with clinicians including nurses, doctors, physiotherapists, quality improvement leads and pharmacist. To maximise experience and role variation of the sample, clinicians were purposively approached by the research nurses in each site, volunteered and consented to take part in the FG. ${ }^{9}$ Participants volunteered and consented prior to the FG. Each FG lasted 60-90 min and was recorded using a digital voice recorder. The interview guide was reviewed by the research team to assess clarity of the questions prior to the FG (online supplementary material).

Two qualitative nurse-researchers (KK and JH), external to any of the participating ICUs, facilitated the FG and recorded reflective notes during and after to adjust for bias in the interpretation of findings. A summary of the discussion was provided at the end of each FG and participants confirmed the data. The audio data were transcribed verbatim and inserted in NVivo V.10 for data management. The transcripts were returned to one participant from each FG to comment on the accuracy of the data.

Data were analysed using an inductive approach to developing themes combining analytical strategies from applied thematic analysis, ${ }^{10}$ and grounded theory. ${ }^{11}$ Data analysis started as soon as the first interviews were transcribed. The transcripts were read and reread independently by the lead qualitative researcher (KK) and an independent qualitative researcher $(\mathrm{GH})$, who created and defined codes. The preliminary coding schema was discussed, revised and verified before all data within each theme were examined and agreed to by the research team (KK, GH, JH and TSW). We developed a codebook to maintain consistency, to allow comparisons, and ensure that the data themes were clearly based on the relationships and linking across the different codes derived from the transcripts. Our approach to analysis was iterative and discussions on analysis and theorising continued throughout the study. We achieved data sufficiency, ${ }^{12}$ and thematic saturation when we observed a rapid decrease in code development and increase in the frequency of assigned codes and themes after five FG were analysed. We continued with the data collection because we needed to have a detailed exploration of sedation practice in all participating ICUs. A final report was prepared and approved by one of the participants in each ICU for accuracy of the data. We used the consolidated criteria for reporting qualitative research checklist when writing our report. ${ }^{13}$

\section{Patient and public involvement}

Patients were not involved in these FG, and we did not consider necessary to conduct a patient and public involvement consultation as the focus of the study was on clinicians' perceptions.

\section{FINDINGS}

\section{Demographics}

The characteristics of each ICU are presented in table 1 . Forty-eight clinicians participated in eight FG, with a range of roles and working ICU experience from 2 to 33 years (table 2).

Three themes emerged from the inductive analysis: (a) a recent shift in sedation practice, (b) uncertainty in decision-making and (c) system-level factors including the ICU environment, organisational factors and educational gaps (figure 1).

\section{A recent shift in sedation practice}

Clinicians recognised the benefit of sedation holds and reflected on the challenges in sedation practice experienced in the past 10 years.

SeniorNurse1: When I started in ICU all those years ago, everybody was paralysed.

Consultant: I think there has been an improvement in the ventilator bundle type thing, people have realised that patients are okay, when they start to breathe spontaneously, and can be restarted on a smaller dose of sedation later... It's been deemed acceptable to do this, whereas before it was considered to be inhumane stopping sedation.

Nurse1: Yes, now you're getting your regular sedation breaks. [ICU5]

There was agreement that optimum sedation was challenging in achieving a balance between wakefulness of the patient and safety (patient being awake, calm, comfortable, 
Table 1 Description of participating Scottish ICU, including size and case mix

\begin{tabular}{|c|c|c|c|}
\hline \multirow[b]{2}{*}{ ICU } & Characteristics of ICU & \multirow[b]{2}{*}{ Type of ICU } & \multirow[b]{2}{*}{ Type of ICU } \\
\hline & $\begin{array}{l}\text { No of beds/ICU beds-HDU } \\
\text { beds }\end{array}$ & & \\
\hline (ICU 2) & 9 beds/ICU & ICU & $\begin{array}{l}\text { Upper gastrointestinal surgery, colorectal surgery, } \\
\text { emergency surgery, medical emergencies. }\end{array}$ \\
\hline (ICU 3) & 18 beds/11 ICU -7 HDU & Mixed & $\begin{array}{l}\text { General medical, surgical, liver transplant, cardiac } \\
\text { patients, trauma, paracetamol overdose disease, } \\
\text { psychiatric patients, drug abusers. }\end{array}$ \\
\hline$(\mathrm{ICU} 4)$ & 7 beds/ICU & ICU & $\begin{array}{l}\text { Medical, surgical, trauma, single-organ failure, } \\
\text { paediatrics, neurological-neurosurgical. }\end{array}$ \\
\hline$(\mathrm{ICU} 7)$ & 20 beds/12 ICU $-8 \mathrm{HDU}$ & Mixed & $\begin{array}{l}\text { Surgical (elective and emergency), medical, centre } \\
\text { for pancreatic patients, burns, plastics and upper } \\
\text { gastrointestinal surgery, complex orthopaedic pelvic } \\
\text { and orthopaedic tumour surgery. }\end{array}$ \\
\hline (ICU 8) & 6 beds/ICU & ICU & $\begin{array}{l}\text { Surgical, medical, alcohol and drug overdoses, cardiac } \\
\text { arrests, non-invasive ventilation. }\end{array}$ \\
\hline
\end{tabular}

ICU, intensive care unit; HDU, high dependency unit.

haemodynamically stable, pain free and safe with reduced risk of adverse events, such as pulling out endotracheal tube or invasive lines). An optimally sedated patient was a patient intubated, sitting up reading the paper (ICU2, doctor). For the nurse in ICU4, the aim in sedation care was not necessarily to achieve optimum sedation; but optimum patient comfort (ICU4, senior nurse). Suboptimum sedation meant both oversedation and undersedation. An oversedated patient was more challenging to recognise and was characterised by a negative symptom, covert and not always easily recognisable.

Consultant2: It's like why a patient presents to the hospital more often with a heart attack quickly than a stroke; because a stroke is a negative symptom. A heart attack gives you pain, so people are far more likely to react to a positive symptom, like climbing out of the bed, than somebody who is flat.

Consultant3: But the risks of over-sedation are kind of more difficult to see and more hidden.

Nurse: Yes, I mean definitely if they are not breathing and triggering their own ventilation, then they are over sedated [ICU3].

Undersedation presented with agitation, distress, ventilator and breathing dyssynchrony, or a fierce look as if they've got wild eyes (ICU1, nurse), and was overt when sedation was reduced or stopped.

Although clinicians had a good understanding of these signs and symptoms, they perceived the recent evolution in practice demanding for two main reasons: (a) there was a change in the use of sedative agents with inconclusive evidence for their effectiveness, and (b) the "just sedate less' approach mandated by the protocols was not applicable to all patients.

\section{A change in the use of sedative agents}

The sedation-analgesia agents for each ICU are presented in table 3. Both nurses and doctors showed a preference towards an intermittent mode of agent administration.

Consultant: Things that have changed in the last couple of years, a movement away from Midazolam and Morphine, has certainly happened since I've started in the unit.

Nurse: I've hardly ever seen it and I started six years ago.

Consultant: I would like to see us to move away from increasing the rate towards giving a bolus [ICU2].

The challenge was observed in the case of managing delirious and agitated patients. The inconclusive evidence and the increased costs of some agents did not support a standardised approach and allowed clinical preferences and trialand-error behaviours, in particular with new agents.

Consultant: The group that are the problem are those who, with acceptable doses of these drugs, they get other stuff. So, you have somebody who gives them benzos and someone else comes along and gives haloperidol, some allow prozine. So, they end up with 


\begin{tabular}{|c|c|c|}
\hline ICU & $\begin{array}{l}\text { No of } \\
\text { participants }\end{array}$ & Experience \\
\hline (ICU 1) & 7 & $\begin{array}{l}2 \text { senior nurses (>9years) } \\
1 \text { nurse (<6years) } \\
1 \text { physiotherapist } \\
1 \text { specialist medical trainee } \\
1 \text { consultant }\end{array}$ \\
\hline (ICU 2) & 6 & $\begin{array}{l}1 \text { senior nurse ( }>10 \text { years) } \\
1 \text { nurse }(<6 \text { years) } \\
1 \text { Scottish Patient Safety } \\
\text { Programme link nurse ( }>10 \text { years) } \\
2 \text { physiotherapists } \\
1 \text { consultant }\end{array}$ \\
\hline (ICU 3) & 6 & $\begin{array}{l}1 \text { senior nurse }(>10 \text { years) } \\
1 \text { nurse }(<6 \text { years) } \\
1 \text { advanced nurse practitioner } \\
\text { (ANP) ( }>10 \text { years) } \\
1 \text { physiotherapist } \\
2 \text { consultants }\end{array}$ \\
\hline (ICU 4) & 6 & $\begin{array}{l}3 \text { senior nurses (>10years) } \\
1 \text { physiotherapist } \\
1 \text { specialist medical trainee } \\
1 \text { consultant }\end{array}$ \\
\hline (ICU 5) & 7 & $\begin{array}{l}3 \text { senior nurses (>8years) } \\
2 \text { nurse }(<6 \text { years) } \\
1 \text { physiotherapist } \\
1 \text { consultant }\end{array}$ \\
\hline (ICU 6) & 3 & $\begin{array}{l}2 \text { senior nurses (>18year) } \\
1 \text { physiotherapist }\end{array}$ \\
\hline (ICU 7) & 7 & $\begin{array}{l}3 \text { senior nurses (>9years) } \\
2 \text { specialist medical trainees } \\
1 \text { consultant } \\
1 \text { pharmacist }\end{array}$ \\
\hline (ICU 8) & 6 & $\begin{array}{l}3 \text { senior nurses ( }>13 \text { year) } \\
2 \text { physiotherapists } \\
1 \text { ANP }\end{array}$ \\
\hline
\end{tabular}

ICU, intensive care unit.

multiple things that are given together. I'm not being critical. It's our fault that we are not having a more standardising way forward. And that's the group that you get them through the 'wild eye' out to the far end.

SeniorNurse1: But that can lead to the other end of the game as well, because until the last few years, we never had any chemical drug that we are familiar with to use apart from the big heavy sedatives. Perhaps we haven't been really up to date with our drug practice. [ICU1]

Below, the clinicians commented on the new agent dexmedetomidine, which has recently been introduced.

SeniorNurse1: It is something, we have used, but it doesn't seem to fit what it was designed to do, what it has been licensed for.
Pharmacist: We probably haven't tried it on enough patients yet. Well in the studies, they didn't use it in difficult to control patients.

SeniorNurse2: I know, the study it was used in was in all comers and it showed a marginal improvement, whereas it's been licensed for difficult patients. [ICU7]

Deviation from 'just sedate less' approach

Clinicians in all ICUs identified deviations in the use of existing sedation hold guidelines and bundles of care (table 3 ). The majority of clinicians adjusted the sedation using a trial-and-error approach, due to the heterogeneity of patient cases and the lack of prescriptive sedation approaches, which allowed the use of clinical preferences and judgement.

Nurse1: It's all individual.

Consultant: It is individualised to the patient but not by any discreet parameters, because some people do it by certain, rigid parameters... Like how fast they are breathing, how much oxygen they are on, how much PEEP ... we don't really do that. [ICU5]

SeniorNurse2: Sometimes we would review it and we would say we'll stop the Propofol but leave the morphine running and things like that, so we have to judge that on the patients themselves.

ANP: We tend to try different things for different people, and I think we find that a bit more workable. [ICU8]

\section{Uncertainty in decision-making}

Specific clinical conditions, namely patients with head injuries, substance withdrawal syndromes and psychotic patients, proved challenging in sedation management and were described as difficult to sedate because, when awakened, they presented with symptoms of agitation, violence and aggression. Ambiguity in identifying the source of agitation (ie, pain, delirium and discomfort), and the unpredictability of the patient's altering consciousness level were barriers to deciding on the appropriate sedation-analgesia management.

MedicalTrainee1: Those that are chronic drug users, they are quite hard to manage, because they'll take you to use second-line medication ... it is hard to wean them, to get a balance, to get them to the point that you can extubate them safely.

Consultant: And the underlying condition is going to make them delirious or they are demented, or both, so you've got to make sure you've got not too heavy a hammer but you may need a degree of chemical restraint in order to keep things okay until their brain failure achieves its best recovery. [ICU7]

MedicalTrainee: I think what we haven't cracked is, what we do with people who A doesn't work, B doesn't work or C doesn't work. And, maybe next door (other patient) it does...I don't know. 


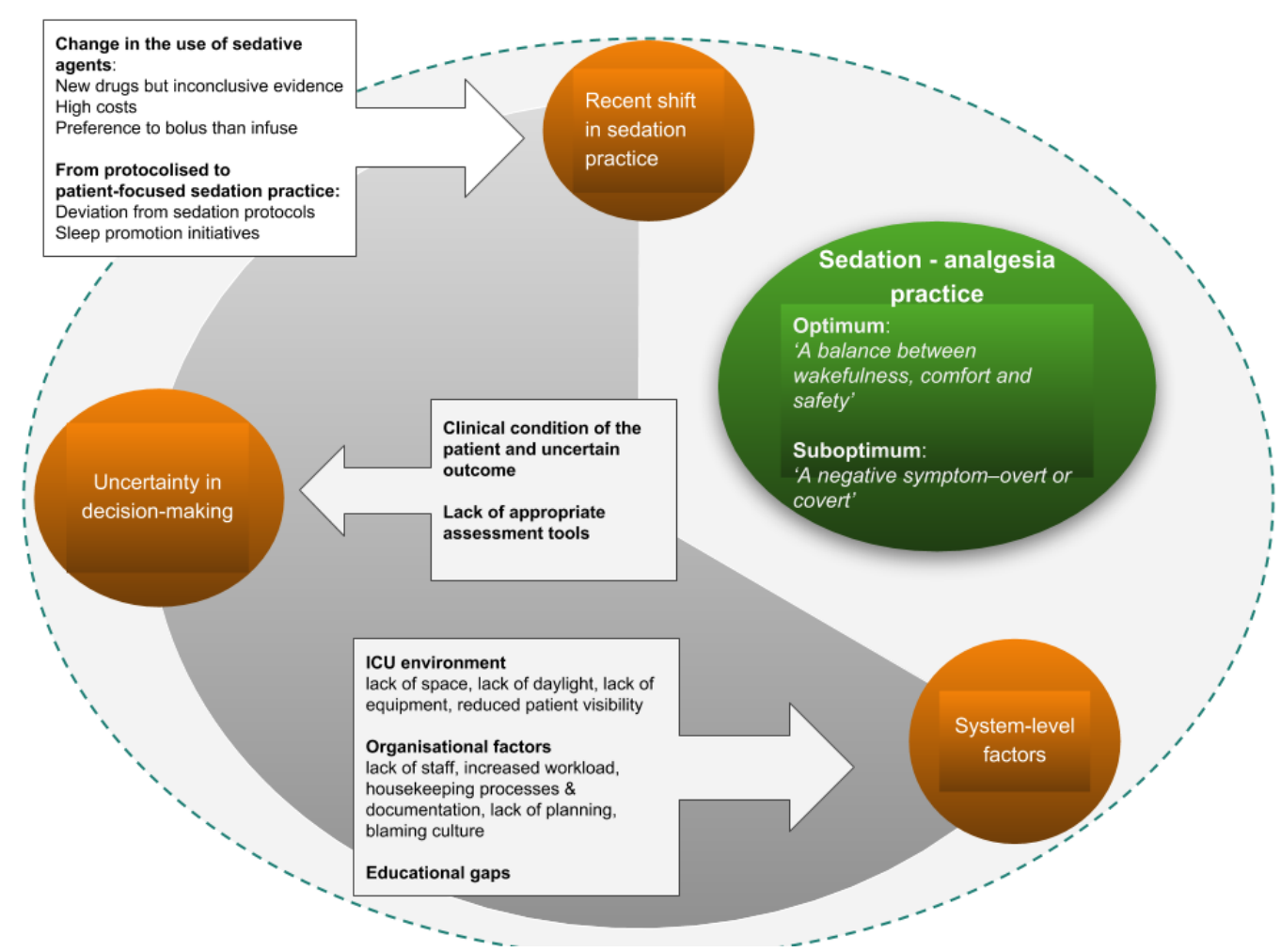

Figure 1 Themes derived from the FGs. Sedation-analgesia practice, in oval, is the principal topic. The main barriers to optimum sedation-analgesia practice are presented in circles with explanatory information in the arrow boxes, and within a pie shape border to symbolise their obstructive role-. FG, focus groups; ICU, intensive care unit.

Nurse: I think it is still very early for that. I think we are finding our feet with it. [ICU1]

Patients admitted with substance withdrawal symptoms during their ICU stay was a common phenomenon, particularly in four ICUs (5, 6, 7 and 8). Although guidelines for the management of substance withdrawal syndromes were available in three out of the four ICUs, nurses used their clinical judgement to manage agitation, and performed sedation holds selectively considering their confidence to ensure that the patient remains safe. In five of the ICUs that admitted patients with an underlying mental condition, clinicians kept the patients sedated until the patient was ready for extubation and commonly increased sedation to manage agitation overnight to ensure patient safety.

SeniorNurse1: If you don't want to stop their sedation because you've got to help elsewhere, because, when they come round, they'll be agitated.

ANP: There's a difference at night as well, like you're stopping sedation during the day, quite a lot of times they ask you to increase it again at night because of agitation, and sometimes to keep them safe. [ICU8]

The lack of appropriate assessment tools, in particular for pain, to identify the source of agitation contributed to clinicians' uncertainty. In most ICUs, clinicians followed a trial-and-error approach to analgesia management. For the physiotherapists, pain management prior to treatment was important to ensure patient compliance, and so nurses ensured comfort by administering common prescribed analgesics.

Physiotherapist2: If they're sore, can't have physio because they are too sore and...

SeniorNurse1: So, we'll try this and see how they are and sometimes give them IV Paracetamol or give them boluses as opposed to continuous infusion. [ICU6]

\section{System-level factors}

\section{ICU environment}

Lack of space and the artificial ICU environment were considered barriers in four of the ICUs (4, 5, 6 and 8). A small ICU, with lack of windows to allow day-light in, and the lack of equipment for patient mobilisation did not facilitate time orientation and early mobilisation for the ICU patients. The consultants in ICU4 stated that patients are too close to each other and their psychological wellbeing can be affected by an agitated patient who is at the next bed space. There is no sense of calmness that would allow the patient to relax. On the other hand, large ICUs had reduced visibility of patients and reduced proximity to observe the agitated patients.

SeniorNurse: You've just got to shout really loudly to get help and we do have individual cubicles which are quite isolated, so we try not have agitated patients within there. 


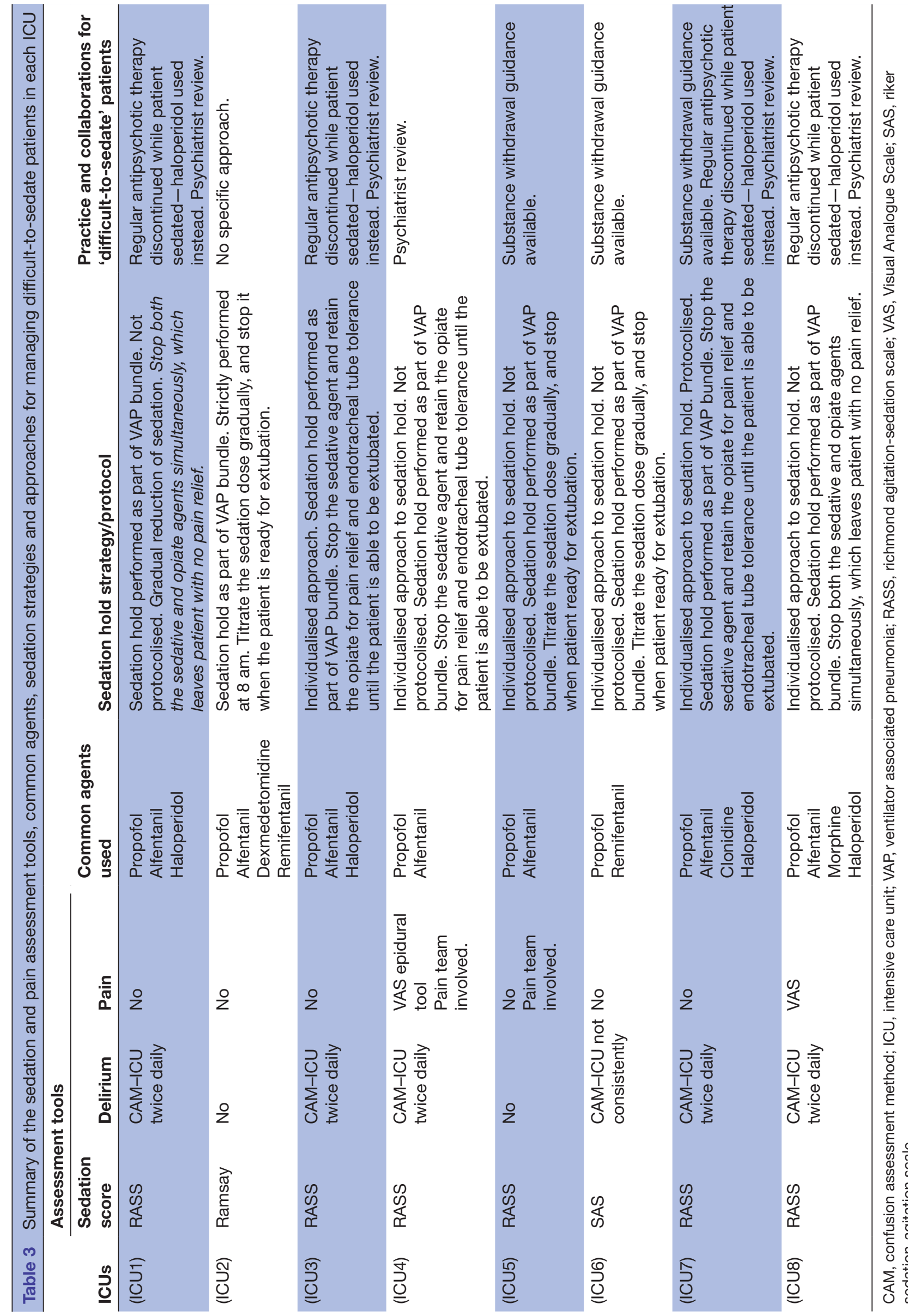

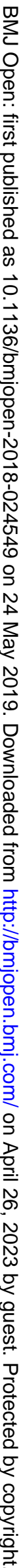


Consultant: And also, just the architectural design, our visibility, there are concrete pillars in front of them, so these are issues which alter the ability to communicate. [ICU7]

Sleep promotion initiatives, advocated in the sedationanalgesia guidelines, were considered challenging in all ICUs, due to the busy ICU environment. Although clinicians adapted the environment to create a day-night effect (ie, reduce the noise and artificial lighting), these changes were not feasible always. Yet, increasing sedation overnight to promote sleep was common in five ICUs $(1,3,5,7$ and 8$)$.

Consultant2: The other thing that I am very guilty of, is giving patients a quiet night. And I equate sedation to sleep, because I kind of go, its night time they should be asleep.

Nurse: You know when you get admissions at midnight, one in the morning and ... all of the lights are on or everything is happening. [ICU3]

\section{Organisational factors}

\section{Lack of staff and increased workload}

Availability of clinical support staff, who would be able to cover patient care during break periods and assist with early mobilisation was not achievable always due to the lack of staff and the increased workload, despite the 1:1 nurse:patient ratio. Appropriate skill mix and availability of senior nurses in each shift was important to ensure support during enactment of sedation holds. Nurses established that when the workload was increased with new admissions or interventions, they tended to increase sedation or delay sedation holds to ensure patient safety.

...just before Christmas there were a couple of days we just couldn't do sedation holds on patients, because there wasn't physically staff here. (ICU7, SeniorNurse)

\section{Housekeeping processes and documentation}

Clinicians referred to housekeeping processes as the daily processes, usually in the form of checklists, to ensure consistency and compliance with care bundles and guidelines. Sedation holds, as part of the ventilator associated pneumonia care bundle, were documented daily in medical notes. There were opposing views regarding the rigidity of implementing these care bundles and becoming a tick box exercise that reduced autonomy in nurses' role. A common concern for nurses was the synchronised conduct of sedation holds in all patients at the start of the day shift, and the challenge of managing agitation as a result of this.

Nurse: After the wash, after breaks when you have got a good couple of hours with your patient and you can just slowly reduce it or stop it; rather than worrying that you are going to go for your morning break and leave a nurse struggling with your patient and their patient.
Consultant2: I think if at the end of the day, it has not been tried, it's unacceptable. But I think you are right, we need to change our culture; if you can justify why, you should just do it when it is suitable. [ICU3]

Both nurses and medical staff were in favour of an individualised, holistic and target-driven approach to sedation practice that would provide an explicit plan of sedation management with set daily goals and feedback on patient progress, and would promote communication among clinicians. Currently, this did not happen in most ICUs, which increased ambiguity about the care of the patient, and allowed debates and inconsistencies between the nurses and the medical staff. Documentation of care plans with prescribed sedation dosages and levels, and plans for failure would encourage nurses to act autonomously and support their decisions. Yet, this was not standardised within the housekeeping processes and hindered information flow and communication among clinicians.

Consultant1: The other bit that kind of gets me and I am probably pretty guilty of this; is failure to plan for failure. Because, I will say 'great wean them off' but I don't actually say what happens if you don't wean them. You guys probably feel a bit unsupported.

Nurse: The thing is when I have asked to re-adjust the dosage, nobody has ever denied it to me and said 'oh no he can't go up'. [ICU3]

\section{Blaming culture}

A blaming culture was a barrier to effective teamwork; a view that was shared among clinicians in four of the ICUs $(1,3,5$ and 7$)$. Nurses felt they lacked support from both the senior nurses and the medical colleagues in relation to sedation management. Being blamed and challenged in the case of an adverse event (eg, unplanned extubation) made them feel disempowered. Issues of trust between nurses and doctors were raised.

Nurse: No-one believes you when you say they (the patient) tried to punch me.

Physiotherapist: I would agree...if somebody dislodges a tracheostomy, you do feel a bit guilty. [ICU3]

\section{Educational gaps}

Clinicians considered the lack of continuous development and education a barrier to their optimum sedation-analgesia practice. Due to financial restraints, education coordinators who could support nurses' educational needs were available only in two ICUs ( 6 and 7 ). In the remaining six, senior charge nurses had this role within their remit. When specifically asked about educational gaps, clinicians identified the need to focus on the management of suboptimum sedation, the use of assessment tools, sedation approaches for specific clinical conditions, such as psychiatric patients and a broader understanding of pharmacokinetics and pharmacodynamics. 
Consultant: So there is a huge problem for education. People sometimes have to take their own time to be able to educate; and nurses don't get that time.

Nurse 1: ... the only education that we have to do, is to try and sort it ourselves. [ICU5]

\section{DISCUSSION}

This standalone study examined clinicians' identified challenges in managing sedation-analgesia in eight Scottish ICUs in the era of a progressive mantra of 'just sedate less' imposed by guidelines, ${ }^{1}$ and identified more similarities than differences. The in-depth understanding of the contextual factors that influence sedation-analgesia practice informed the proposed implementation strategies and the design of some interventions (ie, education package) to be used by the Scottish ICUs involved in the development and evaluation of strategies to improve sedation quality in intensive care quality improvement trial. Although factors such as the unpredictability of the patient's clinical condition and behaviour when awakened, the inconclusive evidence on sedation-analgesia agents and the imposed use of sedation strategies have previously been reported in studies, ${ }^{14}$ this study highlighted that the lack of appropriate assessment tools to recognise the source of agitation, and the lack of sedation-analgesia planning of care increased clinicians' uncertainty of sedation-analgesia management. Our study confirmed that system-level factors including the ICU environment, increased workload, lack of staff, a blaming culture and obstructive daily processes have been reported previously to hinder staff communication, workflow and teamwork. ${ }^{15} 16$

This study highlighted that the rigidity of sedation strategies and lack of adaptable sedation-analgesia planning created tension for clinicians who supported an individualised approach to sedation-analgesia management. Most previous trials that have used daily sedation holds/ protocols support that the effectiveness of these interventions is uncertain and probably context specific, ${ }^{17-20}$ as they do not target to change the clinicians' behaviour in sedation management. Conversely, a context-specific approach to sedation-analgesia practice that improves communication, autonomy, planning of care and focuses on behaviour change will more likely advance the existing sedation-analgesia management. Being aware of such contextual factors could assist in the design of future interventions to improve sedation-analgesia quality.

Attention was paid to the management of pain and discomfort, and promoting sleep, which was highlighted as an area for further development. Recent work ${ }^{21}$ has demonstrated a substantial reduction in ICU length of stay (LOS), mortality, need for ventilator support by using an integrated approach to pain, delirium and agitation. This study confirmed that assessment of suboptimum sedation proved difficult, as discomfort, pain were rarely assessed and usually with inappropriate or poorly validated tools, which may further complicate the patient's condition $^{22}$ and reduce patient-staff communication. ${ }^{23}$ Sleep promotion strategies have been advocated in a number of intervention studies, ${ }^{24-26}$ although a recent Cochrane review, ${ }^{27}$ on non-pharmacological approaches to sleep promotion found low quality of evidence and stressed the need for further high-quality research. The question raised by participants is whether future interventions should embed a holistic approach to improve sedation-analgesia quality, rather than simply achieve compliance with sedation protocols and guidelines.

Finally, the lack of ongoing education on sedation-analgesia care and quality has previously been reported. ${ }^{28} \mathrm{In}$ Scotland in the last 10 years, there has been a significant reduction in educational support for critical care nurses due to financial constraints in NHS, which leaves critical care nurses unsupported and less prepared to meet the challenges of sedation-analgesia management.

There are limitations to this qualitative study. First, it was conducted in eight Scottish ICUs and the findings represent the perceptions of the participants involved. We did not collect information about age or socioeconomic background of the clinicians as we did not intend to make a correlation analysis of the findings. We did not aim to generalise our findings to ICUs at national or international level, as they are context specific; yet, we achieved an in-depth understanding of the challenges in sedation-analgesia practice in Scotland. Second, we used a mixed experience and role sample of professionals in the FG, which might have risked junior staff's ability to express their perceptions freely. Credibility was enhanced by including a variety of ICUs across one country and by using purposive sampling to produce maximum variation in the sample and reduce selection bias. However, we acknowledge that only one pharmacist was involved in our sample and so pharmacists' perspective was under-reported. We did not conduct individual interviews because we aimed to identify the dynamics in each ICU, although we acknowledge the limitation in examining independent perspectives. To reduce the unavoidable risk of bias by the interviewers' presence, increase the rigour of the design and ensure triangulation, the transcripts were reviewed by participants, and the coding scheme was examined by the independent researcher and the research team. We increased dependability by conducting all eight FG, transcribing and analysing them within a short timespan.

\section{CONCLUSION}

This study informed the current literature on the challenges of mandating less sedation and emphasised the need for considering those in the design of future interventions that aim to improve sedation-analgesia quality. Such interventions should engage staff in the change to enable behaviour change, to raise awareness about clinical performance and generate quality improvement initiatives that could contribute to optimising sedation-analgesia.

Acknowledgements The trial was funded by a grant from the Chief Scientists Office, Scotland (CZH/3/3) and with an unrestricted grant from GE Healthcare. 
Collaborators Anaesthetics, Critical Care and Pain Medicine (Prof T S Walsh MD, KK PhD, K Everingham PhD, E C Phillips MBChB), Edinburgh Clinical Trials Unit (JA BSc, J Stephen PhD), Centre for Population Health Sciences (R J Lee MSc, Prof C J Weir PhD), University of Edinburgh, Edinburgh, Scotland, UK; Edinburgh \& Edinburgh Napier University, Scotland, UK (KK PhD, JH PhD); Edinburgh Health Services Research Unit, Edinburgh, Scotland, UK (JH, Prof C J Weir); GE Healthcare Finland Oy, Kuortaneenkatu 2, 00510 Helsinki, Finland (K Uutela PhD, P Peltola BN); Department of Anaesthetics, Ninewells Hospital, NHS Tayside, Scotland, UK (S Cole FFICM); Department of Anaesthetics, Glasgow University, Glasgow Royal Infirmary, Glasgow, Scotland, UK (T Quasim MD); Department of Anaesthetics, Monklands Hospital, NHS Lanarkshire, Scotland, UK (J Ruddy FFICM); Department of Anaesthetics, Victoria Hospital, Kirkcaldy, NHS Fife, Scotland, UK (M McDougall FRCA); Department of Anaesthetics, Victoria Infirmary, NHS GGC, Glasgow, Scotland, UK (A Davidson FFICM); Department of Anaesthetics, Dumfries and Galloway Royal Infirmary, NHS Dumfries and Galloway. Scotland, UK (J Rutherford PhD) and Department of Anaesthetics, Forth Valley Royal Hospital, NHS Forth Valley, Scotland, UK (J Richards FFICM). The DESIST investigators: ROYAL INFIRMARY EDINBURGH: Prof TSW, Dr Alasdair Hay (PI), Dr KK, Fiona Pollock, Louise Boardman, Corrienne McCulloch, Heidi Dawson, David Hope, Dr Kallirroi Kefala, Dr Michael Gillies, Louise Bell, Deborah Rodgers, Sue Wright, Dr Kirsty Everingham, Dr Emma Phillips. DUMFRIES AND GALLOWAY ROYAL INFIRMARY: Dr John Rutherford (PI), Dr Dewi Williams, Catherine Jardine. GLASGOW ROYAL INFIRMARY: Dr Tara Quasim (PI), Dr Alex Puxty, Steven Henderson, Naomi Hickey, Elizabeth Lennon, Jane Ireland, Natalie Dickinson, Marie Callaghan, Dominic Rimmer. VICTORIA INFIRMARY, GLASGOW: Dr Alan Davidson (PI), Katherine McGuigan, Anissa Benchiheub, Laura Rooney. FORTH VALLEY ROYAL HOSPITAL: Dr Jonathan Richards (PI), Janice Grant, Pamela Scott, Marianne Mallice. VICTORIA HOSPITAL, KIRKCALDY: Dr Marcia McDougall (PI), Claire McGinn, Sarah Gray, Keith Boath, Louise Doig, Lesley Berry, Edward Greenwood, Elish Daglish, Carolyne Bullions, Elaine Black, Donna Beattie, Elaine Paton, Alison Connelly, Nancy Hudson, Neville Tomkins, Julia Cook, Terry Hughes, Lynne Cairns, Jennifer Rowe, Ben Slater, Susan Russell, Bob Savage, Gavin Simpson, Ben Shippey. NINEWELLS HOSPITAL, DUNDEE: Dr Stephen Cole (PI), Louise Cabrelli, Jackie Duffy, Pauline Amory. MONKLANDS HOSPITAL: Dr James Ruddy (PI), Margaret Harkins, Elizabeth Reaney, Lyndsey Kearney, Angela Hamill, Isobel Paterson. EDINBURGH CLINICAL TRIALS UNIT: JA (Trial Manager), Ronald Harkess, Samantha Thomas. STATISTICAL TEAM: Dr Christopher Weir, Robert Lee, Jacqueline Stephens. GE HEALTHCARE: Petra Peltola, Kimmo Uutela, Lasse Kamppari, Mika Sarkela. LEARNPR0 (Education Module): Christine Blaydon, Shaun McWhinnie. Edinburgh Health Services Research Unit: Dr Janet Hanley. Independent Data Monitoring Committee: Prof Danny McAuley (Chair); Prof John Norrie, Dr Stephen Wright.

Contributors TSW was chief investigator, had the original idea for the study, secured funding, designed the project and contributed to study management and analysis. KK designed the qualitative process evaluation, and contributed to study management, data collection and analysis. JA contributed to study design and study analysis, was trial manager. JH contributed to study design, data collection and analysis. GH contributed to study analysis. All the authors reviewed and approved the final manuscript.

Funding The DESIST study was funded by a grant from the Chief Scientists Office, Scotland (Ref $\mathrm{CZH} / 3 / 3$ ), and by an unrestricted grant from GE Healthcare.

Competing interests GE Healthcare partly funded the DESIST study, as unrestricted support, but had no control over research design, data analysis or interpretation, manuscript writing or the decision to publish this study.

Ethics approval Ethical approval was secured by Scotland A Research ethics committee.

Provenance and peer review Not commissioned; externally peer reviewed. Data sharing statement № additional data are available.

Open access This is an open access article distributed in accordance with the Creative Commons Attribution Non Commercial (CC BY-NC 4.0) license, which permits others to distribute, remix, adapt, build upon this work non-commercially, and license their derivative works on different terms, provided the original work is properly cited, appropriate credit is given, any changes made indicated, and the use is non-commercial. See: http://creativecommons.org/licenses/by-nc/4.0/.

\section{REFERENCES}

1. Barr J, Fraser GL, Puntillo K, et al. Clinical practice guidelines for the management of pain, agitation, and delirium in adult patients in the intensive care unit. Crit Care Med 2013;41:263-306.
2. Aitken LM, Bucknall T, Kent B, et al. Protocol-directed sedation versus non-protocol-directed sedation to reduce duration of mechanical ventilation in mechanically ventilated intensive care patients. Cochrane Database Syst Rev 2015;1:Cd009771.

3. Mehta S, Burry L, Fischer S, et al. Canadian survey of the use of sedatives, analgesics, and neuromuscular blocking agents in critically ill patients. Crit Care Med 2006;34:374-80.

4. Egerod I. Cultural changes in ICU sedation management. Qual Health Res 2009;19:687-96.

5. Tanios MA, de Wit M, Epstein SK, et al. Perceived barriers to the use of sedation protocols and daily sedation interruption: a multidisciplinary survey. J Crit Care 2009;24:66-73.

6. Payen JF, Chanques G, Mantz J, et al. Current practices in sedation and analgesia for mechanically ventilated critically ill patients: a prospective multicenter patient-based study. Anesthesiology 2007;106:687-95.

7. Walsh TS, Kydonaki K, Antonelli J, et al. Rationale, design and methodology of a trial evaluating three strategies designed to improve sedation quality in intensive care units (DESIST study). BMJ Open 2016;6:e010148.

8. Walsh TS, Kydonaki K, Antonelli J, et al. Staff education, regular sedation and analgesia quality feedback, and a sedation monitoring technology for improving sedation and analgesia quality for critically ill, mechanically ventilated patients: a cluster randomised trial. Lancet Respir Med 2016;4:807-17.

9. Barbour R. Introducing qualitative research. a student's guide. $2^{\text {nd }}$ ed UK: Open University, 2014.

10. Guest G, Namey EE, Mitchell M. Qualitative research: defining and designing. In: Guest G, MacQueen K, Namey E, eds. Applied thematic analysis. Thousand Oaks, CA: Sage, 2012:1-40.

11. Charmaz K. Constructing grounded theory. Los Angeles: Sage, 2014.

12. Dey I. Grounding grounded theory. San Diego: Academic Press, 1999.

13. Tong A, Sainsbury P, Craig J. Consolidated criteria for reporting qualitative research (COREQ): a 32-item checklist for interviews and focus groups. Int J Qual Health Care 2007;19:349-57.

14. Subramanian $P$, Allcock N, James V, et al. Challenges faced by nurses in managing pain in a critical care setting. J Clin Nurs 2012;21(9-10):1254-62.

15. Everingham K, Fawcett $\mathrm{T}$, Walsh $\mathrm{T}$. 'Targeting' sedation: the lived experience of the intensive care nurse. J Clin Nurs 2014;23:694-703.

16. Rose L, Fitzgerald E, Cook D, et al. Clinician perspectives on protocols designed to minimize sedation. $J$ Crit Care 2015;30:348-52.

17. Rose RL, Bucknall T. Staff perceptions on the use of a sedation protocol in the intensive care setting. Aust Crit Care 2004;17:151-9.

18. Mehta S, Burry L, Cook D, et al. Daily sedation interruption in mechanically ventilated critically ill patients cared for with a sedation protocol: a randomized controlled trial. JAMA 2012;308:1985-92.

19. Gill KV, Voils SA, Chenault GA, et al. Perceived versus actual sedation practices in adult intensive care unit patients receiving mechanical ventilation. Ann Pharmacother 2012;46:1331-9.

20. Miller MA, Bosk EA, Iwashyna TJ, et al. Implementation challenges in the intensive care unit: the why, who, and how of daily interruption of sedation. J Crit Care 2012;27:218.e1-218.e7.

21. Mansouri P, Javadpour S, Zand F, et al. Implementation of a protocol for integrated management of pain, agitation, and delirium can improve clinical outcomes in the intensive care unit: a randomized clinical trial. J Crit Care 2013;28:918-22.

22. Woien H, Stubhaug A, Bjork IT. Analgesia and sedation of mechanically ventilated patients - a national survey of clinical practice. Acta Anaesthesiol Scand 2012;56:23-9.

23. Ely EW, ShK I, Bernard GR, et al. The validity and reliability of the Confusion Assessment Method for the Intensive Care Unit (CAMICU). JAMA 2001;286:2703-10.

24. Eliassen KM, Hopstock LA. Sleep promotion in the intensive care unit-A survey of nurses' interventions. Int \& Crit Care Nurs 2011:27:138-42.

25. Elliott R, McKinley S. The development of a clinical practice guideline to improve sleep in intensive care patients: a solution focused approach. Intensive Crit Care Nurs 2014;30:246-56.

26. Engwall M, Fridh I, Johansson L, et al. Lighting, sleep and circadian rhythm: An intervention study in the intensive care unit. Int \& Crit Care Nurs 2015;31:325.35.

27. Hu RF, Jiang XY, Chen J, et al. Non-pharmacological interventions for sleep promotion in the intensive care unit. Cochrane Database Syst Rev 2015;6:CD008808.

28. Hellyer TP, Ewan V, Wilson P, et al. The Intensive Care Society recommended bundle of interventions for the prevention of ventilatorassociated pneumonia. J Intensive Care Soc 2016;17:238-43. 